\title{
Importancia de la motivación socio-afectiva de maestros a estudiantes durante la práctica pedagógica en las diferentes áreas del saber ${ }^{1}$
}

\section{Importance of the socio-affective motivation of teachers to students during the pedagogical practice in the different areas of knowledge}

\author{
DOI: http://dx.doi.org/10.17981/cultedusoc.9.3.2018.29
}

Artículo de investigación. Fecha de recepción: 15/06/2018. Fecha de aceptación: 27/11/2018

\author{
Massiel Navarro Orozco ${ }^{2}$ y Pierina Cantillo Cera ${ }^{3}$ \\ IED de Salamina (Colombia) \\ navarromassiel20@hotmail.com
}

Para citar este artículo:

Navarro, M. y Cantillo, P. (2018). Importancia de la motivación socio-afectiva de maestros a estudiantes durante la práctica pedagógica en las diferentes áreas del saber. Cultura. Educación y Sociedad 9(3), 255-262. DOI: http://dx.doi.org/10.17981/cultedusoc.9.3.2018.29

\section{Resumen}

La presente propuesta surge de una investigación sobre la estrategia didáctica psicopedagógica para la innovación de la práctica docente en la Institución Educativa Departamental de Salamina - Magdalena, ante la necesidad de innovar las practicas docentes en las instituciones educativas. Teniendo en cuenta la importancia de la relación existente entre el docente y el estudiante, su práctica en el aula y su relación con lo socio afectivo para diseñar y caracterizar su labor. La metodología utilizada fue de tipo cualitativo y abarca principalmente la investigación como estrategia pedagógica IEP, desde un diseño descriptivo. Se utilizaron técnicas como: observación participante, el diario de campo y entrevistas como herramienta para la recolección de datos. Se realizó un muestreo intencional o de conveniencia, escogiendo los estudiantes que tenían dificultades en lectura y escritura por cada curso, la muestra estuvo conformada por doscientos treinta (230) estudiantes con edades que oscilaron entre los cuatro (4) y doce (12) años, de grados comprendidos entre preescolar y quinto de primaria. Se logró evidenciar que mediante la relación profesor-estudiante se puede lograr intervenir de manera positiva en el correcto desarrollo del aprendizaje, motivando habilidades investigativas por medio de las tabletas; herramientas TIC didácticas, elevando así la calidad de la educación y el clima escolar.

Palabras clave: Convivencia, Motivación, innovación, socio-afectivo.

\section{Abstract}

The present proposal arises from a research on the psychopedagogical didactic strategy for the innovation of the teaching practice in the Departmental Education Institution of Salamina - Magdalena, before the need to innovate the teaching practices in the educational institutions. Taking into account the importance of the relationship between the teacher and the student, their practice in the classroom and their relationship with the affective partner to design and characterize their work. The methodology used was of a qualitative nature and mainly covers action research, from a descriptive, exploratory design. Techniques were used such as: observation, participants and field diary. An intentional or convenience sampling was carried out, choosing students who had difficulties in reading and writing for each course, the sample consisted of two hundred and thirty (230) students with ages ranging between four (4) and twelve (12) years, of grades between preschool and fifth grade. It was finally shown that through the teacher-student relationship, it is possible to intervene in a positive way in the correct development of learning, thus raising the quality of education and the school climate.

Keywords: Coexistence, Motivation, innovation, socio-affective.

1Este artículo ha sido derivado del Programa de Fortalecimiento de la Cultura Ciudadana y Democrática CT+I a través de la IEP apoyada en TIC en el Departamento de Magdalena: CICLON

2 Líder del Grupo de Investigación "Las Just Do It" de la IED de Salamina.

3 Docentes miembros del Grupo de Investigación "Las Just Do It" de la IED de Salamina.

- The author; licensee Universidad de la Costa - CUC.

Cultura, Educación y Sociedad vol. 9 no. 3, pp. 255-262. Diciembre, 2018

Barranquilla. ISSN 2389-7724 Online 


\section{Introducción}

El presente trabajo de investigación surge como respuesta a la necesidad latente en la Institución Educativa Departamental de Salamina, la cual no cuenta con un bienestar estudiantil de la mano de un equipo interdisciplinario para satisfacer las necesidades pedagógicas afectivas y de convivencia que tanto necesitan los estudiantes que albergan las sociedades en estudio.

Con el apoyo que brinda el departamento de orientación escolar a la escuela hay alta probabilidad de que se minimicen los problemas didácticos, de cobertura y calidad. Para ello, se requiere que el gobierno nacional cubra la necesidad de las escuelas del departamento del Magdalena, poniendo a su disposición un equipo interdisciplinario.

Esta investigación sería una alternativa que permitirá implementar estrategias que mejoren los ambientes de aula y que a su vez lleve a mejorar la disciplina y los saberes, ya que desde el servicio de orientación y asesoría escolar, como organismo especial que ayuda a los docentes en la tarea de instruir, educar y orientar a los estudiantes, tratando siempre de atender a sus necesidades, a su vez le facilita el trabajo al docente en la tarea de individualizar la atención para cada uno de los alumnos que tiene a su cargo, se contribuye a mejorar las prácticas de aula.

Educar, desde la escuela infantil a la secundaria, los componentes y procesos psicológicos necesarios para desarrollar unas relaciones sociales e interpersonales competentes y respetuosas con los demás es un objetivo hoy preferente en el intento de prevenir los problemas de violencia que aquejan a los centros escolares y a la sociedad, en general, la educación tradicional se ha interesado en enseñar lo cognitivo con olvido de lo socio- afectivo y emocional. Hoy en día se comprende mejor la idea que no solo se debe enseñar lo cognitivo, sino también hacer énfasis en el desarrollo social y emocional (Trianes y García, 2002).

Una de las principales razones por las que los estudios desarrollados en los últimos tiempos abarcan con gran importancia temas relacionados a la educación y a la forma de recibir la misma, se debe al nivel tan alto de fracaso escolar, o a su vez el abandono de las escuelas por parte de los estudiantes (Maquillon, 2011).

Las estrategias socio-afectivas de aprendizaje han sido estudiadas por diversos investigadores. Este hecho revela la importancia y relevancia que posee el conocimiento acertado de cómo se pueden aplicar las estrategias socio-afectivas en el proceso de aprendizaje de diferentes áreas a trabajar. No obstante, estas estrategias han sido aplicadas mayormente en situaciones de aprendizaje de mayor nivel, entrando en temas más profundos y de mayor importancia (Rosas, 2007).

Los escenarios de violencia en la sociedad actual requieren con urgencia desarrollar procesos socio-afectivos; pues precisamente son los sentimientos y las emociones puntos focales en la formación de valores (Fernández, Luquez, Leal, 2010). La empatía y la motivación que se tiene al momento de buscar los recursos para llegar a la meta y cumplir cada uno de los logros propuestos son las dos competencias de la inteligencia emocional que intervienen en el éxito de las relaciones socio- afectivas (Morales, 2012).además, se recomienda que el espacio y el ambiente donde de la escuela donde se desarrolla el niño, sea de un clima favorable, tanto emocional como pasivo para facilitar así, el desarrollo 
socio-afectivo del estudiante (Tejada, 2010).

Por cada maestro en el mundo que promueve una muy buena relación socioafectivo con sus alumnos, existen cinco que no lo hacen de forma correcta, o que simplemente no lo hacen, es decir que el nivel promedio de maestros se ubica en la categoría de ineficacia a nivel afectivo, lo cual evidentemente provoca sufrimiento en los alumnos (Madrigal, Mujica, Tamarín, Olave y Carrasco, 2011).

El estudiante, al momento de desear aprender algún área durante su periodo de estudio, necesita estar en un ambiente que lo motive, una de las principales motivaciones para un estudiante seria su maestro, quien debe actuar como guía y apoyar al alumno en situaciones de dificultad, si el estudiante no encuentra ese apoyo en su maestro muy difícilmente podrá comprender de manera acertada el tema a estudiar (Gil, Blanco y Guerrero, 2005). De esta manera se observa que en la situación de cumplimiento, los profesores están más prontos a percibir el éxito de logro de aprendizaje escolar, como producto directo de sus esfuerzos como agentes motivacionales (Castro, Jofré, Vega y Bortoluzzi, 2012).

En la actualidad existe el consenso casi unánime de que los factores afectivos influyen en la adquisición de conocimientos, por parte del estudiante, la motivación o afecto que este reciba de las personas a su alrededor influyen directamente en la motivación e inspiración, sin embargo, la investigación de tales variables resulta complicada, especialmente debido a cuestiones de identificación y medición (Barrios, 1997).

El ambiente educativo no se limita a las condiciones materiales necesarias para la implementación del currículo, también es importante destacar las relaciones interpersonales básicas entre maestros y alumnos y las acciones involucradas a experiencias y vivencias por cada uno de los participantes de tal manera que sea reflejada las acciones socio-afectivas (Duarte, 2003).

Se considera establece un tipo de relación de cooperación entre los alumnos, que estimula su desarrollo cognitivo y socio-afectivo, lo que resulta imprescindible para el aprendizaje de actitudes y valores socialmente valiosas (Ferreiro, 2007). Los estilos de aprendizaje desde una óptica holística, que supere la visión eminentemente cognitivista que ha prevalecido en su estudio en el marco de la Psicología Educativa, han permitido desarrollar ideas que hasta ahora son insuficientemente valoradas, como lo es el abordaje de los estilos de aprendizaje, y haciendo énfasis en las relacionadas con la dimensión socio-afectiva del proceso de aprendizaje (Blanco y Guerrero, 2011).

La influencia sobre el proceso de socialización de las relaciones que se establecen entre los alumnos, así como su importancia para el logro de los objetivos socio-afectivos, es un hecho reconocido desde muchos años atrás, pero los efectos de estas relaciones sobre el aprendizaje escolar son un aspecto mucho más discutido, quizá esto se deba a las consecuencias que pueda traer el modelo pedagógico que contempla al profesor como principal agente educativo (Coll, 1984).

Cuando el análisis se centra en los chicos y se analiza su comportamiento en función de su rendimiento académico se comprueba que del total de estrategias Metacognitivas los buenos alumnos son más eficaces que los malos en la frecuencia de uso de las estrategias de Autoconocimiento, lo que supone que la intervención del maestro eta ligada pero no de un cien por ciento, puesto que 
la actitud del alumno como primera impresión tiene gran valor al momento de desarrollar estrategias que contribuyan a un mayor aprendizaje (Lozano, González, Núñez, Lozano y Álvarez, 2001).

Esta investigación sería una alternativa que ante múltiples inquietudes se hace necesario implementar para mejorar los ambientes de aula, para que de esta manera puedan mejorar la disciplina y los saberes, ya que desde el servicio de orientación y asesoría escolar, como organismo especial que ayuda a los docentes en la tarea de instruir, educar y orientar a los estudiantes, tratando siempre de atender a sus necesidades, a su vez le facilita el trabajo al docente en la tarea de individualizar la atención para cada uno de los alumnos que tiene a su cargo, se contribuye a mejorar las prácticas de aula.

\section{Motivación socio-afectiva del maestro al alumno}

Se entiende que la interacción del profesor con sus alumnos, conlleva procesos afectivos, un docente, más que un guía durante el desarrollo intelectual, debe sentir cierto afecto por el alumno para poder contribuir de manera asertiva a su desarrollo, lo que generaría un clima social en el aula, y propicio para el aprendizaje, esto plantea la necesidad de describir y analizar las dimensiones afectivas de la docencia que están presentes durante la interacción en el aula, así como su impacto sobre el aprendizaje de los estudiantes (García, 2009).

En los últimos tiempos se han escuchado voces que aluden a ciertos cambios en las actitudes, percepciones y prácticas de los docentes, vinculando ese movimiento con una nueva o distinta realidad escolar. Nuevos escenarios sociales y culturales se "imponen" en las escuelas, a pesar de ser éstas instituciones fuertemente conservadoras, muchos docentes "profesionales" no encuentran la manera correcta de enseñar, consideran que el simple hecho de dictar una clase es lo que responde a su trabajo, pero, hay que tener en cuenta que al tratar con personas, en este caso estudiantes, más que seres que tienen la necesidad de aprender requieren de atención y motivación, dichos requisitos deberían ser brindados por el maestro durante el desarrollo de la clase. Al no ser así, la respuesta del aprendizaje por parte de los estudiantes se verá fracasada (Alliaud, 2004).

Los mecanismos comunicativos que emplean los docentes para facilitar y favorecer el aprendizaje de los alumnos y promover el establecimiento de un clima de aula emocionalmente positivo requiere de varios aspectos (Cuadrado y Fernández, 2008).

- analizar los comportamientos comunicativos, verbales, no- verbal y prosódico que profesores y maestros emplean en el aula.

- conocer si existen diferencias en dichos comportamientos discursivos entre maestros y profesores.

- caso de existir diferencias, averiguar si la formación psicopedagógica inicial que recibieron los maestros es un factor decisivo que dé respuestas a las mismas.

\section{Metodología}

Se realizó un estudio de tipo cualitativo, utilizando el modelo de investigación acción participante (IAP), fundamentada en la línea metodológica de la investigación como estrategia pedagógica (IEP) (Mejia, 2011), desde un diseño descriptivo. La investigación cualitativa busca conocer e interpretar la realidad de los participantes a través de sus propias experiencias, 
entregando una información subjetiva del fenómeno de estudio. (Hernández, Fernández, y Baptista, 2010). Por su parte el modelo de investigación acción participante plantea una producción de conocimiento basado en la reflexión de los participantes, teniendo en cuenta la participación activa de maestros en el proceso de enseñanza y acompañamiento tecnológico, guiando al estudiante en su proceso de investigación. Generando nuevo saber y conocimiento sobre una realidad determinada en un proceso de aprendizaje colaborativo.

Los estudios con un diseño descriptivo están encaminados a especificar las características de la población sujeto de estudio o los fenómenos que sean sometidos a análisis (Hernández, Fernández, y Baptista, 2010). En cuanto a la población beneficiada con el estudio, se tomó una muestra representativa de la comunidad estudiantil de doscientos treinta (230) estudiantes de la Institución Educativa Departamental de Salamina - Magdalena, distribuidos en los cursos desde preescolar hasta quinto de primaria, con edades que oscilaron entre los cuatro (4) y doce (12) años, se realizó un muestreo de tipo intencional, escogiendo los estudiantes que presentaban características relacionadas con el objeto de estudio.

Para la recogida de datos se utilizó la observación participante, técnica de recolección cualitativa que implica que el docente tome un rol activo en el proceso de investigación; asimismo, se utilizó el diario de campo, donde se registran y sistematizan los elementos obtenidos de la observación participante donde el contacto con las personas del contexto, modulan las reflexiones y conclusiones que se pueden extraer. (Ramos, 2013) y entrevistas, debido a que no solo debe estar como espectador del proceso de implementación, sino, que también debe participar del mismo, registrando cada elemento observado con relación a la variable de estudio.

La metodología del proyecto de investigación se realizó a partir de los recorridos de las trayectorias de indagación, basados en la integración de la investigación como estrategia pedagógica (IEP) apoyada en TIC al aula, articulado al plan de estudios, transversalizando así los procesos de lectura y escritura a todas las áreas del conocimiento, lo anterior con la finalidad de generar una trasformación social y educativa a través del aprendizaje en contexto, involucrando intereses, motivaciones y realidades de los estudiantes para generar conocimiento científico, donde se establecieron cinco (5) trayectos planteado por la IEP que especifican la ejecución y las metas trazadas para darle solución a la pregunta problema planteada. Los trayectos utilizados se presentan a continuación.

Trayecto (1): los docentes realizaron un diagnóstico, según los criterios de evaluación de las pruebas por competencias para identificar las dificultades específicas que presentaba la población en los procesos de motivación socio- afectiva

Trayecto (2): diseño de estrategias pedagógicas basadas en la investigación y las TIC transversales a las diferentes áreas de estudio, de acuerdo a las necesidades de la población.

Trayecto (3): implementación y transversalización de las estrategias en el aula de clase por parte del cuerpo docente.

Trayecto (4): análisis e interpretación de los resultados.

Trayecto (5): reflexión y apropiación social sobre los conocimientos generados en materia de estrategias pedagógicas orientadas al aprendizaje y fortalecimiento de procesos motivación socio- afectiva (Mejía, 2011). 


\section{Resultados}

Posterior a un tiempo de observación y realización de entrevistas al personal objeto de estudio, se demostró que la relación socio-afectiva de muchos maestros con sus estudiantes, no es la ideal, y casualmente estos estudiantes eran los mismo que durante tiempos atrás venían presentado dificultades en cuanto a su rendimiento académico e incluso en su relación con los demás compañeros.

En general se puede mencionar que son notarias las falencias imperantes en el ámbito educativo de la institución en estudio, pero con la acérrima voluntad de un equipo de trabajo bien orientado por rector, docente orientador y docente de aulas los resultados tienden a ser mejor en lo instructivo y en lo formativo. Entre los resultados encontrados con la implementación de este proyecto y el metodología de la investigación como estrategia pedagógica (IEP) se logró implementar una estrategia didáctica innovadora interinstitucional, que permita mejorar el ambiente de aula en la institución educativa, de igual manera vincular, prácticas motivacionales, carencias afectivas y ambientes de aula integradas con el PEI de la institución educativa, estimulando de igual manera las habilidades investigativas, permitiendo integrar los conocimiento académicos establecidos por el sistema educativo y los conocimientos de la sociedad, permitiendo integral de manera vivencial los conocimientos que nutren y fortalecen la educación de los estudiantes de la institución (Mejía, 2011). Finalmente resulta relevante destacar que permitió identificar los factores afectivos y motivacionales que influyen en el ambiente de aula, en la institución educativa.

\section{Discusión}

Las relaciones socio- afectivas que tengan los docentes con sus estudiantes, son uno de los temas más estudiados al momento de pensar en la razón por la cual un estudiante no presenta buenos resultados académicos, pues bien, es cierto que la actitud del estudiante frente a la clase influye muchos en temas de desarrollo intelectual, pero la atención y el afecto que este reciba por parte de su docente tendrá también gran influencia, entonces, si en un ámbito escolar más que ponernos a dejar todo el trabajo en manos del alumno, los docentes nos ponemos en marcha para contribuir aún más en su desarrollo, quizá en futuro ya no tengamos que hablar de estudiantes menos intelectuales, pues la actitud del maestro es la principal motivación de un estudiante (Trianes, García, 2002). La acción educativa de la investigación como estrategia pedagógica (IEP) busca promover habilidades investigativas que generen aprendizajes y que estos mismo contribuyan a la construcción de ciudadanos comprometidos, menos excluyente, más democráticos y con sentido de partencia (Mejía, 2011) es decir, el proyecto realizado permite dar un primer paso en el municipio de Salamina, Magdalena, aportando hallazgos que se pueden contrastar en la actualidad con la teoría, pero más adelante se realizaría con otras instituciones, para promover la experiencia de la IEP, en su propuesta metodológica de construir grupos sociales que vivan la práctica educativa y se sientan motivados no solo por sus docentes como guías en el proceso, sino, por ellos mismos como seres capaces, con las herramientas necesarias como lo es la investigación como estrategia para la pedagogía. 


\section{Referencias}

Alliaud, A. (2004). La experiencia escolar de maestros "inexpertos". Biografías, trayectorias y práctica profesional. Revista Iberoamericana de Educación, 34(3), 1-11.

Barrios, M. E. (1997). Motivación en el aula de lengua extranjera. Encuentro. Revista ele Investigación e Innovación en la clase de idiomas, 9.

Castro-Carrasco, P., General, F., Jofré, R., Sáez, N., Vega, Á. y Bortoluzzi, M. (2012). Teorías subjetivas de profesores sobre la motivación y sus expectativas de éxito y fracaso escolar. Educar em Revista, (46).

Coll, C. (1984). Estructura grupal, interacción entre alumnos y aprendizaje escolar. Infancia $y$ aprendizaje, 7(27-28), 119-138.

Cuadrado, I. y Fernández, I. (2008). ¿Cómo intervienen maestros y profesores para favorecer el aprendizaje en secundaria? Un estudio comparativo desde el análisis del discurso. Infancia y Aprendizaje, 31(1), 3-23.

De Tejada, M. (2010). Evaluación de la autoestima en un grupo de escolares de la Gran Caracas. Liberabit, 16(1), 95-103.

Duarte, D. (2003). Ambientes de aprendizaje: una aproximación conceptual. Estudios pedagógicos (Valdivia), (29), 97-113.

Fernández, O., Luquez, P., \& Leal, E. (2010). Procesos socio-afectivos asociados al aprendizaje y práctica de valores en el ámbito escolar. Telos, 12(1).
Ferreiro, R. (2007). Una visión de conjunto a una de las alternativas educativas más impactante de los últimos años: El aprendizaje cooperativo. Revista electrónica de investigación educativa, 9(2), 1-9.

García, B. (2009). Dimensiones afectivas de la docencia. Tema del mes. Revista Digital Universitaria, 10 (11). 1-14.

Gil, N., Blanco, L. y Guerrero, E. (2005). El dominio afectivo en el aprendizaje de las matemáticas. Una revisión de sus descriptores básicos. Revista iberoamericana de educación matemática, 2(1), 15-32.

Lozano, L., González-Pienda, J., Núñez Pérez, J., Lozano, L. y Álvarez, L. (2001). Estrategias de aprendizaje, género y rendimiento académico. $R e$ vista galego-portuguesa de psicoloxía e educación. 5(7). 1138-1663.

Madrigal, C., Mujica, A., Tamarín, C. C., Olave, C. y Carrasco, I. (2011). Clima social escolar en el aula y vínculo profesor-alumno: Alcances, herramientas de evaluación, y programas de intervención. Revista Electrónica de Psicología Iztacala, 14(3), 70-84.

Maquilón, J. y Hernández, F. (2011). Influencia de la motivación en el rendimiento académico de los estudiantes de formación profesional. Revista electrónica interuniversitaria de formación del profesorado, 14(1).

Morales, S. (2012). Estudio del nivel de empatía y motivación de logro de los alumnos de la carrera de odontología de la Universidad de Concepción. Rev. Educ. Cienc. Salud, 9(2), 121125. 
Rosas, E. (2007). Las estrategias socioafectivas y su efecto motivador en situaciones de aprendizaje de una lengua extranjera. Paradigma, 28(2), 181-196.
Trianes, M. y García, A. (2002). Educación socio-afectiva y prevención de conflictos interpersonales en los centros escolares. Revista Interuniversitaria de Formación del profesorado, (44). 